\title{
Progesterone requires heat shock protein 90 (HSP90) in human sperm to regulate motility and acrosome reaction
}

\author{
Vrushali Sagare-Patil $^{1} \cdot$ Rashmi Bhilawadikar $^{2} \cdot$ Mosami Galvankar $^{1} \cdot$ Kusum Zaveri $^{2}$ • \\ Indira Hinduja $^{2}$ • Deepak Modi ${ }^{1}{ }^{\mathbb{C}}$
}

Received: 15 September 2016 / Accepted: 19 January 2017 / Published online: 24 February 2017

(C) Springer Science+Business Media New York 2017

\begin{abstract}
Purpose The aims of this paper were to study whether heat shock protein 90 (HSP90) is a regulator of sperm functions and to determine its association with oligoasthenozoospermia. Methods The levels of HSP90 in sperm lysates were measured by ELISA. Localization of HSP90 and its isoforms was evaluated by immunofluorescence. Sperm motility and kinetics were assessed by computer-assisted sperm analysis. Acrosome reaction was determined by lectin staining.

Results The levels of HSP90 were lower in oligoasthenozoospermic men and correlated positively with the number of motile spermatozoa. In capacitated human spermatozoa, HSP $90 \alpha$ was mostly found in residual nuclear envelope, and the HSP90 $\beta$ isoform was higher in the flagella. Inhibition of HSP90 by geldanamycin or 17-AAG did not affect basal motility, but suppressed progesterone-mediated forward progressive motility, hyperactivation and acrosome reaction. Progesterone treatment dephosphorylated both HSP $90 \alpha$ and HSP90 $\beta$ at Ser/Thr-Pro residues, but not Tyr residues.
\end{abstract}

Capsule This study shows that HSP90 is essential for progesteronemediated sperm motility and acrosome reaction.

Vrushali Sagare-Patil and Rashmi Bhilawadikar contributed equally to this work.

Electronic supplementary material The online version of this article (doi:10.1007/s10815-017-0879-5) contains supplementary material, which is available to authorized users.

Deepak Modi

deepaknmodi@yahoo.com; modid@nirrh.res.in

1 Molecular and Cellular Biology Laboratory, National Institute for Research in Reproductive Health (ICMR), J. M. Street, Parel, Mumbai 400012, India

2 Hinduja IVF Centre, PD Hinduja Hospital and Medical Research Center, Veer Savarkar Marg, Mahim, Mumbai 400016, India
Conclusion HSP90 levels are downregulated in oligoasthenozoospermia, and its functional inhibition attenuates progesterone-mediated sperm motility and acrosome reaction.

Keywords Heat shock protein · HSP90 - Human sperm . Motility · Acrosome reaction · Progesterone ·

Oligoasthenozoospermia $\cdot$ Phosphorylation

\section{Introduction}

Heat shock protein 90 (HSP90) is one of the most abundant and evolutionarily conserved molecular chaperones. Unlike molecular chaperones, HSP90 is not required for de novo protein folding, but it facilitates the final folding of client proteins [1, 2]. The HSP90 client proteins include a range of signal transducers and transcription factors that are essential for diverse cellular functions $[1,2]$. Not surprisingly, any alteration in the activity of HSP90 exerts marked effects on cellular physiology and also causes diseases, including cancers [1-3].

Two major cytoplasmic isoforms of HSP90 exist in eukaryotic cells. Amongst these two, HSP90 $\alpha$ is an inducible form whilst HSP90 $\beta$ is a constitutive form. Arising by gene duplication, HSP90 $\alpha$ and HSP90 $\beta$ differ in their sequences, biochemical nature and molecular functions $[3,4]$. Both these isoforms can form homodimers $(\alpha \alpha$ or $\beta \beta)$ and heterodimers $(\alpha \beta)$, with HSP $90 \alpha$ having a higher propensity to dimerize as compared to HSP90 $\beta$ [3]. Functionally, HSP90 $\alpha$ plays critical roles in multiple cellular processes; HSP90 $\beta$ has a key role in trophoblast differentiation [3].

Beyond somatic cells, HSP90 and its isoforms also play critical roles in male germ cells. During development, HSP $90 \alpha$ is specifically localized in the germ cells of mouse testis; HSP90 $\beta$ is expressed in the somatic cells [5, 6]. Male mice lacking the HSP90 $\alpha$ isoform are infertile owing to a 
block in meiosis [7]. In contrast, mice knockout for HSP90 $\beta$ undergo meiosis; however, these animals develop globozoospermia and a defective acrosome [8]. These observations suggest that there are different functions of HSP90 isoforms in the testis, and they might play independent roles in the regulation of spermatogenesis.

At the biochemical level, in vitro studies have shown a role of HSP90 in the regulation of kinase activity in spermatids. In the mouse spermatids, HSP90 interacts with testis-specific serine/ threonine kinases (TSSKs), which are a family of post-meiotic kinases that are required for sperm maturation [9]. Interestingly, inhibition of HSP90 reduces the activity of TSSKs, leading to its ubiquitination and proteasome degradation [9]. These observations suggest that HSP90 is required in the spermatids to regulate kinase activity, meiosis and sperm maturation.

Whilst the functions of HSP90 in the testis are well elucidated, the role of HSP90 in mature sperm has received little attention. HSP90 is detected in the sperm flagella of multiple species $[10,11]$. During mouse sperm capacitation, HSP90 undergoes tyrosine phosphorylation, and its inhibition augments capacitation [11, 12]. Functionally, HSP90 seems to play a role in the regulation of sperm motility. It has been demonstrated that loss of HSP90 during freezing of bull sperm correlates with a reduction in motility; pharmacological inhibition of HSP90 reduces porcine sperm motility $[13,14]$. Interestingly, inhibition of HSP90 has recently been shown to attenuate progesterone-mediated human sperm hyperactivation and acrosome reaction [15]. These results imply that HSP90 also functions in ejaculated spermatozoa. However, the isoforms of HSP90 required for these functions in human spermatozoa are unknown; its relevance to poor sperm motility warrants further investigations.

In the present study, we aimed to determine the HSP90 isoforms expressed in the human sperm and elucidate its functions.

\section{Material and methods}

The study was approved by the Institutional Review Board and Institutional Ethics Committee on human subjects (project no. NIRRH-103). Informed consent was obtained from all patients.

\section{Normozoospermic and oligoasthenozoospermic samples}

Semen samples were collected by masturbation after 3 days of sexual abstinence. WHO 2010 criteria were used to select the normozoospermic and oligoasthenozoospermic samples [16]. As per these criteria, a sample was considered normozoospermic if it had sperm count $\geq 15$ million/ml and forward motility $\geq 32 \%$. A sample was considered as oligoasthenozoospermic if the sperm count was $<15 \mathrm{million} / \mathrm{ml}$ and $<32 \%$ of spermatozoa had forward motility. Sperm assessment was done by two independent observers, and the diagnosis of oligoasthenozoospermia was confirmed after verification in a second independent sample from the same individual collected a week apart. Spermatozoa were stained with $0.4 \%$ eosin solution and observed under a light microscope to determine the number of round cells. Samples with $>1 \times 10^{6}$ round cells/ml were excluded.

In all, 30 normozoospermic and 30 oligoasthenozoospermic samples were included for measurements of levels of HSP90. The inclusion and exclusion criteria and the clinical parameters of these subjects are reported earlier [17]. There was no history of smoking, alcoholism, cryptorchidism, childhood disease, radiation exposure, prescribed drug usage or presence of varicocele in any of the subjects.

\section{Capacitation and progesterone treatment}

Normozoospermic semen samples were pooled from several anonymized donors and used. Semen samples after liquefaction were centrifuged and the spermatozoa were washed and capacitated for $2 \mathrm{~h}$ in DMEM:F-12 medium (GIBCO, Grand Island, $\mathrm{NY}$ ) containing 2.6\% BSA fraction V (Sigma-Aldrich, St. Louis, MO). Post-capacitation, the spermatozoa were challenged with $5 \mu \mathrm{M}$ progesterone (Sigma) as described earlier [18] and processed for various experiments. This concentration was chosen as previous studies have shown that $5 \mu \mathrm{M}$ progesterone stimulated multiple kinases and increases sperm motility [18].

\section{Immunofluorescence}

Capacitated spermatozoa were fixed in chilled acetone and immunofluorescence was performed as detailed previously [19]. Briefly, cells were blocked in $1 \%$ gelatin, and the slides were incubated overnight with an antibody that recognizes both the isoforms of HSP90 (05-594 Millipore, Temecula, CA). The antibody was used at an optimized dilution of 1:50 in phosphate buffer saline (PBS). For detection of the isoforms, the slides were incubated with an antibody specifically recognizing HSP90 $\alpha$ (07-2174, Millipore) and HSP90 $\beta$ (AB3468, Millipore). Both the antibodies were used at a dilution of 1:100 in PBS. After extensive washings, detection was carried out using appropriate rhodamine-labelled secondary antibody (Jackson ImmunoResearch Laboratories). Cells were counterstained with DAPI (4',6-diamidino-2-phenylindole, Sigma) and mounted in anti-fade (Vector Laboratories, Burlingame, CA). As negative controls, the cells were incubated with donkey serum in place of the primary antibody.

\section{Enzyme-linked immunosorbent assay}

For measurement of HSP90 levels, ELISA was performed as detailed earlier [17]. Briefly, washed spermatozoa were lysed in $2 \%$ SDS and $10 \mu \mathrm{g}$ of protein was coated overnight onto MaxiSorp wells (Nunc, USA). After blocking, the wells were 
incubated with HSP90 antibody (1:50 dilution in PBS) overnight. The wells were washed three times and incubated with horseradish peroxidase (HRP)-labelled secondary antibody. Colour was developed using tetramethylbenzidine (Bangalore Genei, India) as chromogen and $\mathrm{H}_{2} \mathrm{O}_{2}$ as a substrate. All the measurements were done in triplicate. For negative control, the primary antibody was replaced with PBS. For normalization, the levels of $\beta$-actin were estimated in the same protein lysate using the above protocol. The $\beta$-actin antibody (Sigma) was used at a dilution of 1:100 in PBS.

\section{Detection of phosphorylation on HSP90 isoforms}

The levels of phosphotyrosine and phosphoserine/threonineproline residues on HSP90 and its $\alpha$ - and $\beta$-isoforms were determined by ELISA. Goat polyclonal antibodies against total HSP90 and specific to HSP90 $\alpha$ or HSP90 $\beta$ (Millipore) were coated in the wells of a 96-well plate. The wells were blocked with $1 \%$ gelatine (Sigma) and incubated overnight with cell lysates from the control and progesterone-treated samples (10 $\mu \mathrm{g}$ of protein). After extensive washings, the wells were incubated with the monoclonal anti-phosphotyrosine antibody (BD Biosciences, San Jose, CA) or anti-phospho-Ser/Thr-Pro antibody (MPM-2, Millipore) at a dilution of 1:100 (in PBS). After incubation for $2 \mathrm{~h}$ at room temperature and washing to remove excess antibody, the wells were probed with HRPconjugated anti-mouse antibody (Jackson ImmunoResearch Laboratories) at a dilution of 1:20,000 in PBS. Detection and measurements were done as above.

\section{Effect of HSP90 inhibitors}

To study the effect of inhibition of HSP90 activity, capacitated spermatozoa were incubated with $5 \mu \mathrm{M}$ of geldanamycin or 17-AAG (17- $N$-allylamino-17-demethoxygeldanamycin; Invitrogen, San Diego, CA) for $30 \mathrm{~min}$ at $37^{\circ} \mathrm{C}$. This concentration $(5 \mu \mathrm{M})$ of geldanamycin and 17-AAG was chosen as it is known to significantly inhibit HSP90 activity and affect its ability to interact with several client proteins in diverse cell types, including spermatids and sperm [7, 10]. Parallel controls without any treatment or with vehicle were maintained.

After incubation in $5 \mu \mathrm{M}$ geldanamycin or 17-AAG for $30 \mathrm{~min}$, the cells were then challenged with $5 \mu \mathrm{M}$ progesterone for 10 and $30 \mathrm{~min}$. The cells were processed for motility assessment or acrosome reaction, as detailed below. Untreated and progesterone-treated spermatozoa were maintained as controls.

\section{Computer-assisted sperm analysis}

Motility analysis was performed using a computer-assisted sperm analyser (CASA; Hamilton Thorn IVOS V12.3, Beverly, MA) at default settings, as described earlier [18, 20]. Slides were scanned at 60 frames/s to determine the number of motile, progressively motile and hyperactive spermatozoa. Hyperactivation was defined as curvilinear velocity $(\mathrm{VCL})>70 \mu \mathrm{m} / \mathrm{s}$, linearity $(\mathrm{LIN})<65 \%$ and lateral displacement of sperm head (ALH) $>5 \mu \mathrm{m}[18,20]$.

\section{Acrosome reaction}

The number of acrosome-reacted spermatozoa was determined according to the protocol used previously $[18,20]$. Briefly, acetone-fixed spermatozoa were incubated with FITC-labelled lectin from Pisum sativum (Sigma; diluted in PBS in 1:100), counterstained with propidium iodide (Sigma) and observed under a fluorescent microscope (Olympus, Tokyo, Japan). The percentage of acrosome reaction was estimated by counting a minimum of 100 spermatozoa in five biological replicates.

\section{Statistical analysis}

The mean \pm SEM for all the experimental data were computed and statistical analysis was done using GraphPad Prism, version 5, either by Student's $t$ test or by two-way ANOVA using Dunnet's multiple comparison test. The association between the levels of HSP90 and sperm motility was determined by Pearson's $R$ test.

\section{Results}

\section{HSP90 $\alpha$ and HSP90 $\beta$ are differentially localized} in the sperm residual nuclear envelope and flagella

Using an antibody that recognizes both the isoforms, staining for HSP90 was detected in all the spermatozoa at the residual nuclear envelope (RNE), which is at the junction of the head and the midpiece. Immunoreactive HSP90 was also detected in the flagella of nearly $70 \%$ of spermatozoa (Fig. 1). Using an antibody that specifically recognizes HSP $90 \alpha$, staining was detected in the RNE of all the spermatozoa; weak staining was observed in the flagella of $30 \%$ of spermatozoa (Fig. 1). Weak staining for HSP $90 \beta$ was detected in the in the RNE of all the cells; strong staining was detected in the flagella of $\sim 70 \%$ of spermatozoa (Fig. 1). No staining was detected in the negative controls, indicative of the specificity of staining (Fig. 1a).

\section{HSP90 levels are reduced in oligoasthenozoospermic men}

The normozoospermic controls had a sperm count of $51.3 \pm$ $31.2 \mathrm{million} / \mathrm{ml}$ (range $=18-85 \mathrm{million} / \mathrm{ml})$ with progressive motility of $50.2 \pm 7.5 \% \quad(\mathrm{range}=45-58 \%)$. In the oligoasthenozoospermic group, the mean sperm count was 7.5 $\pm 3.8 \mathrm{million} / \mathrm{ml}($ range $=4-12$ million $/ \mathrm{ml})$ and motility was $15.0 \pm 10.9 \%$ (range $=5-25 \%$ ). The mean sperm count and 
a
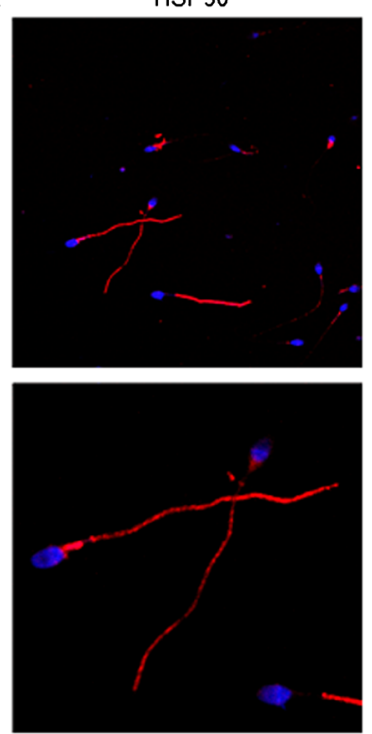

b

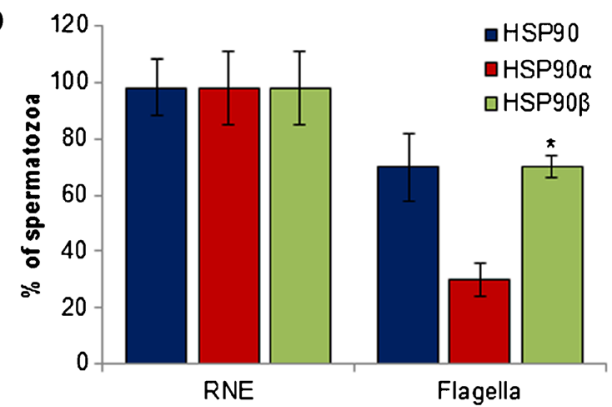

Fig. 1 Localization of HSP90 and its isoforms in human spermatozoa. a Representative immunofluorescence images for HSP90 and its isoforms in human spermatozoa. Capacitated spermatozoa were probed with antibodies against HSP90 (that recognizes both the isoforms) or those that recognize specifically the $\alpha$ - and $\beta$-isoforms. The antibodies were detected using an Alexa 595-labelled secondary antibody. Red fluorescence is staining for HSP90; the nuclei are counterstained with DAPI (blue fluorescence). The negative controls are cells incubated

motility were significantly lower in the oligoasthenozoospermic group as compared to the control.

HSP90 was found to be significantly $(p<0.05)$ reduced in the spermatozoa of oligoasthenozoospermic men as compared to normozoospermic controls (Fig. 2a). A positive correlation between the amounts of HSP90 and the percentage of motile spermatozoa was observed (Fig. 2b). Pearson's correlation coefficient between per cent motile spermatozoa and HSP90 protein level was found to be 0.40 .

\section{Inhibition of HSP90 does not affect basal sperm motility}

The percentages of motile, progressively motile and hyperactive spermatozoa were not significantly different in the control and the 17-AAG- and geldanamycin-treated groups (Fig. 3a). Although geldanamycin treatment marginally decreased the percentage of progressively motile spermatozoa, the effect was not statistically significant $(p=0.35)$. Extending the
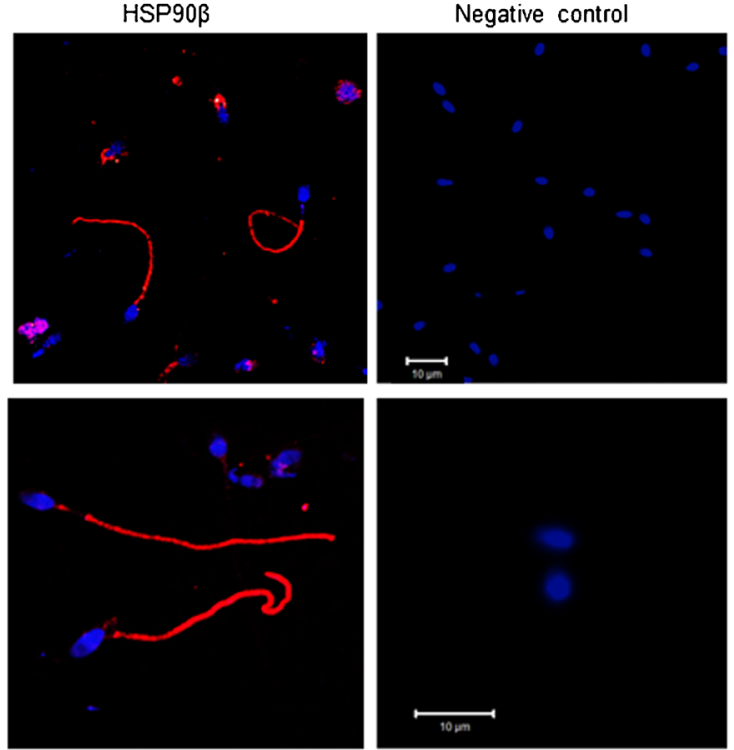

without primary antibody. Upper panel are lower magnification images and lower panel are higher magnification images. Appropriate scale bars are shown in the negative control. The experiment was repeated three times on different pools of semen samples from different individuals. $\mathbf{b}$ Percentages of spermatozoa showing staining in residual nuclear envelope $(R N E)$ and the flagella. Data are the mean $\pm \mathrm{SD}$ for three biological replicates. Asterisk indicates value significantly different as compared to that observed for HSP90

incubation timings or increasing the concentrations of both inhibitors had no effect (not shown). There was no effect of both inhibitors on sperm velocities and other motion kinetic parameters (Supplementary Fig. S1a).

\section{Inhibition of HSP90 attenuates progesterone-driven progressive motility and hyperactivation}

As compared to the controls, progesterone treatment significantly $(p<0.05)$ increased the percentage of motile spermatozoa. This increase in the number of motile spermatozoa was also observed when spermatozoa were incubated with progesterone in the presence of 17-AAG and geldanamycin (Fig. 3b).

The percentage of progressively motile spermatozoa significantly increased upon treatment with progesterone $(p<0.05)$. This increase in the number of progressively motile spermatozoa was not observed when spermatozoa were pre-incubated with 17-AAG and geldanamycin (Fig. 3b). As compared to 

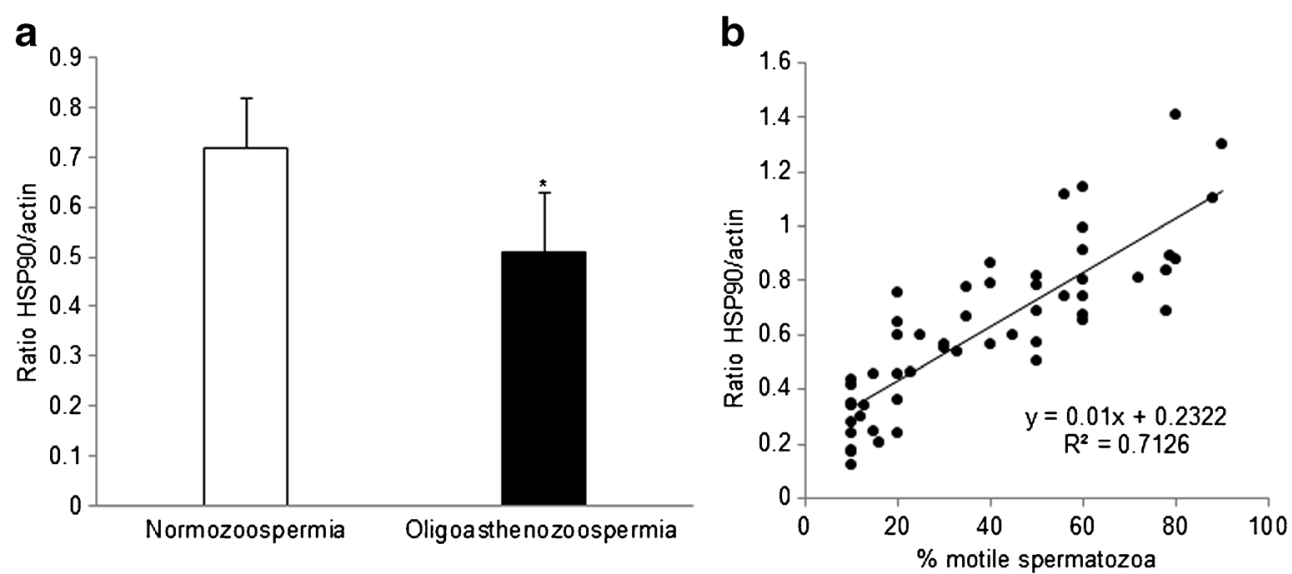

Fig. 2 Association of HSP90 levels and sperm motility. HSP90 levels were measured by ELISA in spermatozoa from normozoospermic and oligoasthenozoospermic men. a Levels of HSP90 in spermatozoa of normozoospermic and oligoasthenozoospermic men. Values on the $Y$ axis are the mean $\pm \mathrm{SE}$ ( $n=30$ in each group) of the ratios of OD at $450 \mathrm{~nm}$ for HSP90/ $\beta$-actin. *Significantly different as compared to

progesterone alone, the percentage of progressively motile spermatozoa was significantly lower when progesterone was given to cells pre-incubated with geldanamycin or 17-AAG $(p<0.05)$.

Progesterone treatment significantly increased the percentage of hyperactivated spermatozoa as compared to untreated controls $(p<0.05)$. This progesterone-mediated increase in hyperactivation was not observed when the spermatozoa were pre-incubated with 17-AAG or geldanamycin (Fig. 3b). As compared to progesterone alone, the percentage of hyperactive spermatozoa was significantly lower when progesterone was given in the presence of geldanamycin or 17-AAG $(p<0.05)$.

The different motion kinetic parameters of capacitated spermatozoa and those treated with HSP90 inhibitors in the presence and absence of progesterone are shown in Supplementary Fig. S1b. As compared to the controls, progesterone treatment significantly increased the average path velocity (VAP), VCL and ALH; the straightness (STR) significantly reduced $(p<0.05)$. This increase in VAP, VCL and ALH and the reduction in STR were not observed in spermatozoa pre-incubated with either geldanamycin or 17-AAG and then treated with progesterone.

\section{HSP90 is required for progesterone-mediated acrosome reaction}

The percentages of spontaneously acrosome-reacted spermatozoa were similar in the controls and geldanamycin- or 17AAG-treated groups. Approximately $20 \%$ of capacitated spermatozoa were found to undergo acrosome reaction with progesterone treatment (Fig. 3c). This fourfold increase in the number of acrosome-reacting spermatozoa in the progesterone-treated group was statistically significant $(p<0.05)$. This increase was not observed when progesterone was administered to spermatozoa pre-incubated with HSP90 normozoospermic men $(p<0.05)$. b Scatter plot for percentage of motile spermatozoa and levels of HSP90 protein. Values on the $Y$-axis are the ratios of OD at $450 \mathrm{~nm}$ of HSP90/actin, and values on the $X$-axis are the percentages of motile spermatozoa. Each spot represents data from one individual

inhibitors. As compared to progesterone alone, the percentage of spermatozoa undergoing acrosome reaction was significantly $(p<0.05)$ lower when treated with progesterone in the presence of geldanamycin or 17-AAG (Fig. 3c).

\section{HSP90 isoforms are dephosphorylated} at the serine/threonine-proline, but not tyrosine, residues in response to progesterone challenge

Total HSP90, HSP90 $\alpha$ and HSP90 $\beta$ were found to be phosphorylated at the tyrosine as well as the serine/threonineproline (Ser/Thr-Pro) residues. The levels of tyrosine phosphorylation of total HSP90 and its isoforms were not significantly different in the control and progesterone-treated cells. As compared to the controls, progesterone treatment decreased the phosphorylation of HSP90 at the Ser/Thr-Pro residues by almost $10 \%$. The phosphorylation of HSP90 $\alpha$ and HSP90 $\beta$ at the Ser/Thr-Pro residues was reduced by almost $25 \%$ in progesterone-treated cells as compared to the controls (Fig. 4). The differences in the levels of Ser/Thr-Pro phosphorylation between the control and progesterone-treated groups were statistically significant $(p<0.05)$.

\section{Discussion}

Heat shock proteins are cytoplasmic chaperons that have wide functions in somatic cells. However, most heat shock proteins are also expressed in spermatozoa [21-23], suggesting their possible role in gamete functions. In the present study, we show that, along with the principal piece, HSP90 is also localized in the junction of the midpeice and the head of capacitated human spermatozoa. Amongst the two isoforms, HSP90 $\alpha$ is dominantly localized in the junction of the head and midpiece; 

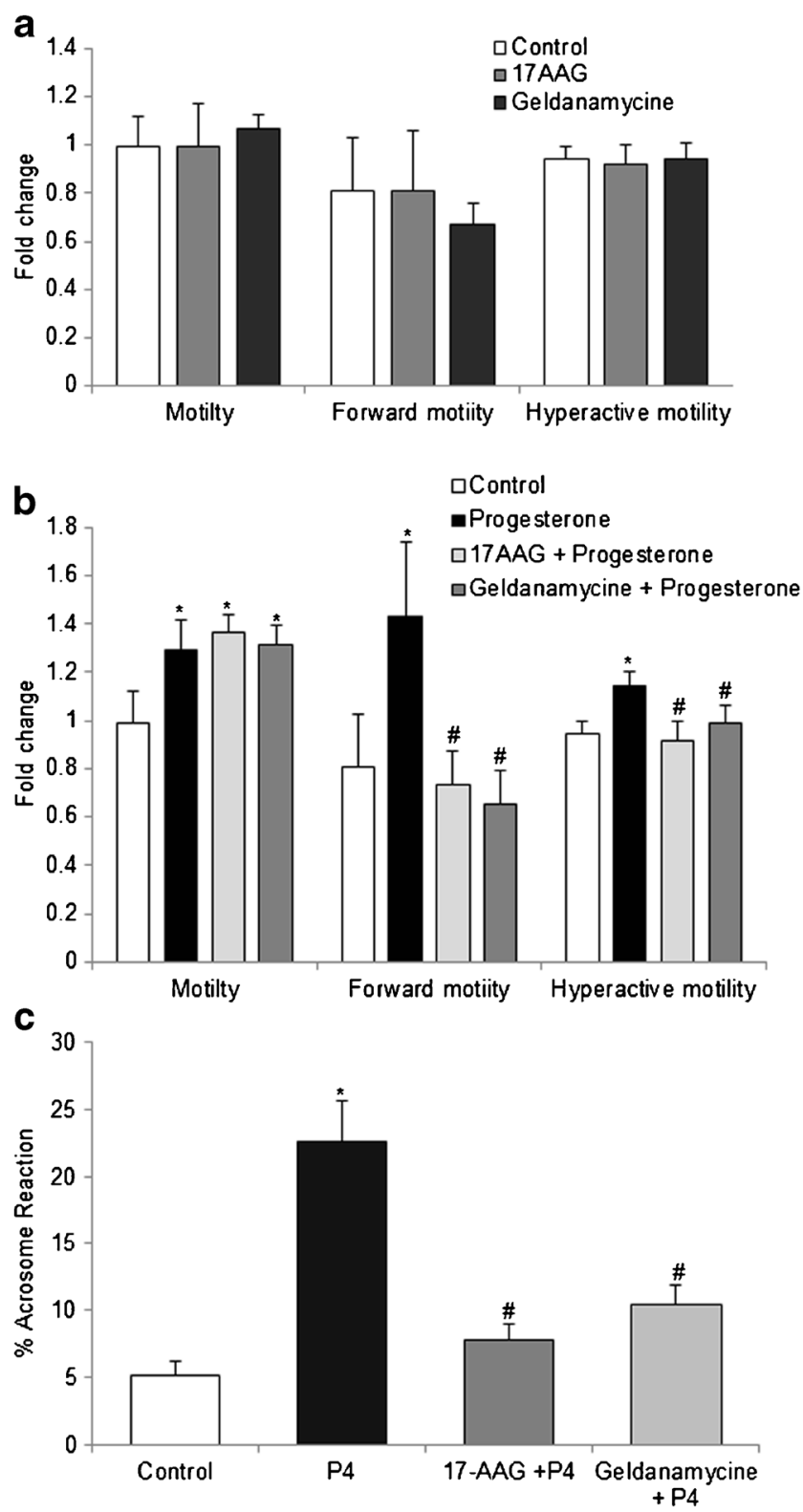

Fig. 3 Effect of HSP90 inhibitors on sperm motility and acrosome reaction. Capacitated spermatozoa were incubated with HSP90 inhibitors (geldanamycin and 17-AAG) with or without progesterone (P4), and motility parameters were assessed by CASA. a Effect of HSP90 inhibitors on basal sperm motility. b Motility pattern in the presence of progesterone. c Percentage of acrosome-reacted spermatozoa as measured by lectin staining. In (a) and (b), values on the $Y$-axis are the mean + SEM of fold change where the value obtained for a normozoospermic sample was taken as 1 . In (c), values on the $Y$-axis are the mean $+\mathrm{SE}$ of the percentages of acrosome-reacted spermatozoa. Data are derived from three independent pools of samples. *Value significantly different as compared to the control. "Value significantly different as compared to progesterone treatment $(p<0.05)$

HSP90 $\beta$ is mainly detected in the tail. To the best of our knowledge, this is the first report demonstrating the isoform-specific differential localization of HSP90 in human sperm. The detection of HSP $90 \beta$ in sperm is surprising as previous studies in the mouse have reported it to be Sertoli cell-specific; HSP90 $\alpha$ is reported to be germ cell-specific $[5,6]$. Whilst this might reflect underlying differences in the requirements of HSP90 in human and mouse sperm, it is noteworthy that mice lacking HSP $90 \beta$ in the germ cells develop globozoospermia [8], implying that the $\beta$-isoform should also be expressed in the germ cells of the testis. It will be of interest to study the developmental expression of HSP90 isoforms in the testis to determine how HSP90 isoforms are regulated during spermatogenesis and when are they acquired in ejaculated sperm.

Quantitatively, the percentages of spermatozoa expressing HSP90 $\alpha$ and HSP90 $\beta$ isoforms in the flagella and the junction of the head and midpiece differ significantly. Whilst both the isoforms of HSP90 are detected in the junction of the head and midpiece, the intensity of HSP90 $\alpha$ is higher than that of HSP90 $\beta$. Interestingly, in the flagella, HSP90 $\beta$ seems to be the major isoform as it was intensely detected in nearly $70 \%$ of spermatozoa; HSP90 $\alpha$ was weakly detected in the flagella and also only in $30 \%$ of spermatozoa. Such disparity in localization and abundance of the two isoforms has not been reported previously and implies that both these isoforms might have different functions in sperm. Whilst proteins in the tail are generally considered to have a role in motility, the functions of the proteins in the junction between the head and midpiece are unclear. The junction of the head and midpiece is termed as the residual nuclear envelope (RNE), which contains cytoplasmic remnants, including the chaperons, and is a site for calcium stores in spermatozoa [24]. It is possible that HSP90 in this region may be a part of the RNE complex of proteins and may participate in sperm calcium homeostasis. Indeed, a recent study has shown that inhibition of HSP90 affects calcium fluxes in capacitating human spermatozoa [15]. Whether this effect is because of inhibition of HSP90 in the RNE needs to be investigated.

Since HSP90 is localized in the sperm flagella, we investigated whether it is required for sperm motility. Asthenozoospermia is a complex condition where majority of spermatozoa in the ejaculate are immotile or poorly motile. A previous study has shown increased phosphorylation of the HSP $90 \beta$ isoform in sperm of men with asthenozoopermia [21]. In the present study, we observed that the levels of HSP90 are significantly lower in the spermatozoa of men with oligoasthenozoopermia as compared to controls. Furthermore, men with greater percentage of progressively motile spermatozoa had higher amounts of HSP90; the levels lowered with a decline in the percentage of motile sperm. Interestingly, in the bull spermatozoa, loss of HSP90 post-freezing correlates with a decrease in motility [12]. These results together imply that HSP90 may be one of the regulators of sperm motility.

To test whether HSP90 is essential for motility, we determined the effects of two specific HSP90 inhibitors, geldanamycin and 17-AAG, on sperm functions. Both these inhibitors have equal affinity for HSP90 $\alpha$ and HSP $90 \beta$ and are known to inhibit HSP90 activity by promoting its degradation. In the present study, we observed that both these 
Fig. 4 Progesterone alters the phosphorylation of HSP90.

Spermatozoa were incubated with or without progesterone for $30 \mathrm{~min}$ and phosphorylation of HSP90 and its isoforms at the tyrosine (Tyr) and serine/ threonine-proline (Ser/Thr-Pro) residues was determined by ELISA. Values on the $Y$-axis are the mean + SE of per cent reactivity with respect to a control (untreated) sample. Data are derived from four independent replicates. *Value significantly different as compared to the control $(p<0.05)$
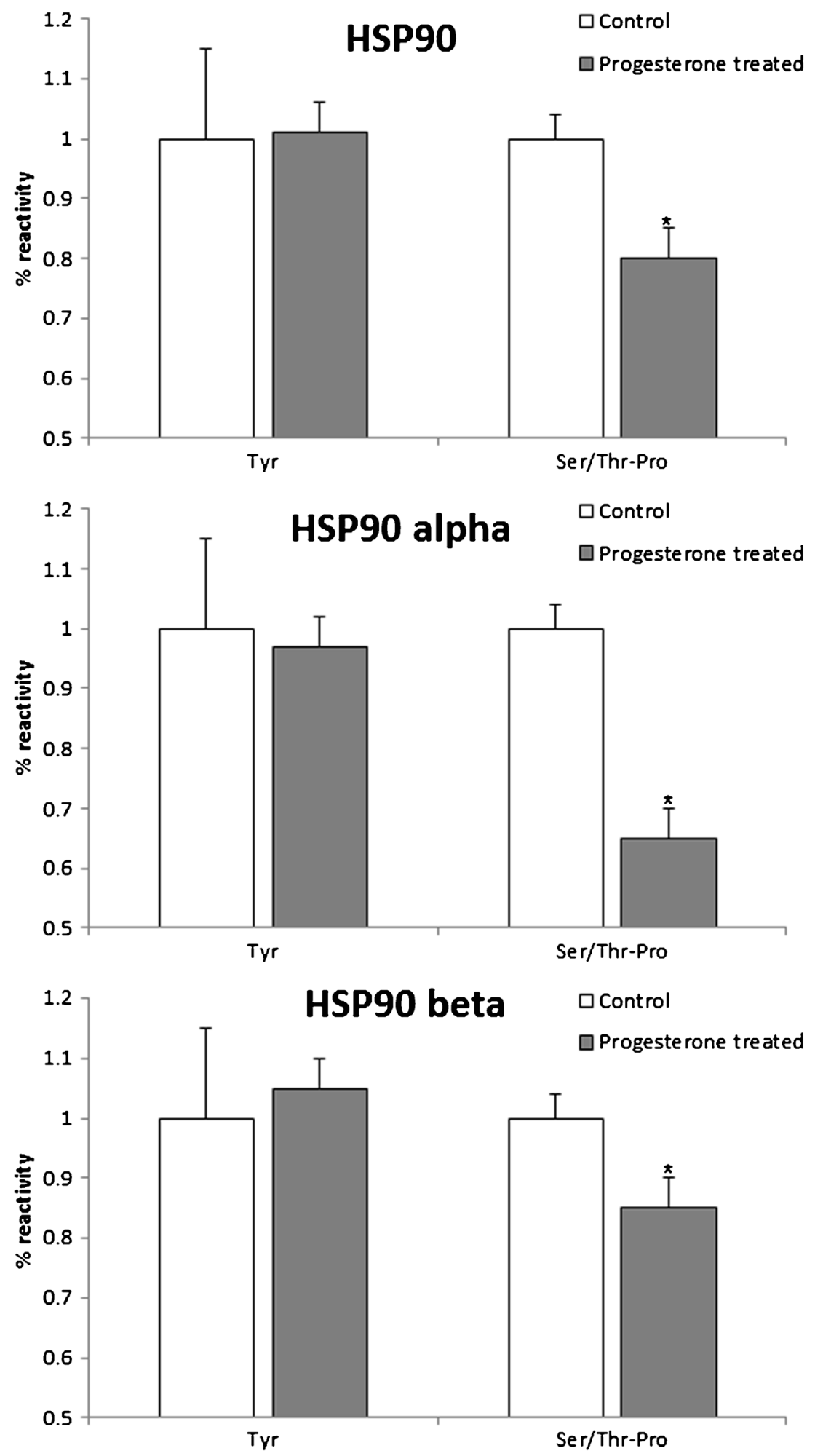

inhibitors of HSP90 did not have any effect on sperm motility or its velocities. In contrast, in the porcine sperm, inhibition of HSP90 by geldanamycin decreases its motility [13]. Differences in the concentrations of the inhibitor used and incongruities in the experimental protocols might be possible reasons for such discrepant findings. However, since two different inhibitors of HSP90 did not affect human sperm motility, unlike the porcine counterparts, basal motility in human sperm does not seem to be HSP90-dependent.

Sperm motility is a complex phenomenon and is stimulated in the female reproductive tract for efficient fertilization. In vitro, this effect can be simulated by treatment of 
capacitated spermatozoa with progesterone, which increases the number of motile spermatozoa and forward progressive motility and induces hyperactivation [18]. Intriguingly, although basal sperm motility was unaffected by HSP90 inhibition, we observed that both inhibitors almost completely abrogated the progesterone-mediated increase in forward progressive motility and hyperactivation. The progesteronemediated increases in VAP, VCL and ALH were significantly reduced by HSP90 inhibition. A similar inhibition of progesterone-mediated increase in hyperactivation by geldanamycin has been recently reported for human sperm [15]. These observations together confirm that HSP90 mediates the responsiveness of progesterone in the regulation of progressive motility and hyperactivation in human spermatozoa. Progesterone-mediated increase in sperm motility is a tightly controlled process and requires activation of several kinases [18-20]. In the mouse testicular spermatids, HSP90 associates with testis-specific kinases which are essential for motility [9]. We have previously shown that progesteronemediated increase in sperm motility also requires the activation of protein tyrosine kinases [18], and interestingly, inhibition of HSP90 blocks the increase in progesterone-mediated tyrosine phosphorylation of sperm proteins [15] (data now shown). Based on these observations, it is alluring to speculate that progesterone may be modifying the activity of HSP90 in spermatozoa to govern the activity of the kinases required for motility. Whether low HSP90 levels in the sperm of oligoasthenozoospermic men result in altered sperm kinase activity leading to blunted responsiveness to progesterone needs to be determined.

Acrosome reaction is a final step that spermatozoa undergo to gain a fertilizing potential. Progesterone present in high concentration in the vicinity of the egg promotes acrosome reaction of the sperm $[18,25]$. Herein, we show that HSP90 is essential not only for progesterone-mediated motility but also induction of acrosome reaction as both geldanamycin and 17-AAG significantly reduced the progesterone-mediated increase in the number of acrosome-reacted spermatozoa. These results are not surprising as inhibition of HSP90 attenuates progesteronemediated increase in calcium influx and tyrosine phosphorylation [15], both of which are required for acrosome reaction [15, 18]. Together, our data suggest that sperm HSP90 is essential for progesterone-mediated signalling events in spermatozoa that culminate in hyperactivation and acrosome reaction.

From the above studies, it is evident that HSP90 plays significant roles in the regulation of sperm functions; however, which isoforms of HSP90 are involved in this process is hitherto unclear. In the absence of inhibitors that can block HSP90 $\alpha$ and HSP90 $\beta$ independently, the isoform-specific functions of HSP90 in spermatozoa are difficult to ascertain by pharmacological approaches. It has been previously reported that the phosphorylation of HSP90 $\beta$ is higher in asthenozoospermic men [21]; we recently reported that progesterone reduces the phosphorylation of HSP90 $\alpha$ in capacitated human spermatozoa [26]. Thus, we argued that if progesterone has a preferential requirement for any one of the two isoforms of HSP90 in the regulation of sperm functions, the phosphorylation of that isoform might differ in response to progesterone treatment. However, to our surprise, we observed that both HSP90 $\alpha$ and HSP90 $\beta$ were dephosphorylated in progesterone-treated spermatozoa, and this dephosphorylation occurred exclusively at the Ser/Thr-Pro residues, but not at the Tyr residues. To the best of our knowledge, this is the first report demonstrating the dephosphorylation of HSP90 isoforms specifically at the Ser/Thr-Pro residues by progesterone. Based on these observations, however, it is difficult to ascertain whether there is any preferential requirement of any one of the HSP90 isoforms in the regulation of sperm functions. Our data do imply that progesteronemediated signalling involves a Ser/Thr-Pro phosphatase that dephosphorylates both the isoforms of HSP90 to elicit its biological response.

How dephosphorylation of HSP90 at the Ser/Thr-Pro residues alters its biological activity in human spermatozoa is difficult to establish. The bioactivity of HSP90 requires the binding of ATP and modulation of the ATP binding pocket of HSP90 $\alpha$, leading to the dissociation of a large variety of client proteins, including kinases $[27,28]$. Interestingly, within this ATP binding pocket of HSP90 $\alpha$, the phosphorylation of Thr-90 and Thr-115/ $425 / 603$ determines its affinity towards different client proteins $[27,28]$. Furthermore, dephosphorylation of HSP90 at specific residues affects its ATPase activity and co-chaperone binding and influences conformational transitions of HSP90 [29]. Thus, the phospho-residues in HSP90 appear as "phosphorylation switch" that modulates its functions to elicit differential biological responses. Intriguingly, both geldanamycin and 17-AAG inhibit HSP90 functions within the same pocket that contains the phospho-residues [30]. Based on these observations, it is tempting to postulate that the phosphorylated form of HSP90 would sequester molecules involved in the regulation of sperm motility and exocytosis; its dephosphorylation by progesterone would lead to switch in its functions, altering its affinity to different client proteins to induce hyperactivation and acrosome reaction. It will be of interest to identify the client proteins of the phosphorylated and non-phosphorylated forms of HSP $90 \alpha$ and HSP90 $\beta$ in spermatozoa to solve the mechanistic basis of how HSP90 would transduce the biologic effects of progesterone and control sperm functions.

In summary, our results have shown that HSP $90 \alpha$ and HSP90 $\beta$ isoforms are expressed in the spermatozoa and their levels are lower in men with oligoasthenozoospermia. We further show that HSP90 is essential for progesterone-stimulated forward progressive motility, hyperactivation and acrosome reaction. We believe that our studies have identified a crucial role of HSP90 in sperm functions. This information might aid in the development of drugs that help enhance sperm motility and, in converse, can be of use in developing sperm-based contraceptives. 
Acknowledgements We are grateful to Dr. A S Sreedhar (Scientist, Centre for Cellular and Molecular Biology, Hyderabad, India) for generously sparing the geldanamycin and 17-AAG used in this study. The study (RA/382/06-2016) is funded by grants from the Department of Biotechnology, Innovative Young Biotechnologist Award (IYBA) and Indian Council of Medical Research (ICMR), Government of India, New Delhi, India. VSP is a recipient of a Senior Research Fellowship from ICMR. DM is a recipient of the IYBA grant.

\section{Compliance with ethical standards}

Conflict of interest The authors declare no conflict of interest.

Funding source Indian Council of Medical Research, Department of Biotechnology, Government of India

\section{References}

1. Li J, Buchner J. Structure, function and regulation of the Hsp90 machinery. Biomed J. 2013;36:106-17.

2. Mayer MP, Le Breton L. Hsp90: breaking the symmetry. Mol Cell. 2015;58:8-20.

3. Sreedhar AS, Kalmár E, Csermely P, Shen YF. Hsp90 isoforms: functions, expression and clinical importance. FEBS Lett. 2004:562:11-5.

4. Taipale M, Jarosz DF, Lindquist S. HSP90 at the hub of protein homeostasis: emerging mechanistic insights. Nat Rev Mol Cell Biol. 2010;11:515-28.

5. Lee SJ. Expression of HSP86 in male germ cells. Mol Cell Biol. 1990;10:3239-42.

6. Vanmuylder N, Werry-Huet A, Rooze M, Louryan S. Heat shock protein HSP86 expression during mouse embryo development, especially in the germ-line. Anat Embryol. 2002;205:301-6.

7. Grad I, Cederroth CR, Walicki J, Grey C, Barluenga S, Winssinger $\mathrm{N}$, et al. The molecular chaperone Hsp90 $\alpha$ is required for meiotic progression of spermatocytes beyond pachytene in the mouse. PLoS One. 2010;5:e15770.

8. Audouard C, Christians E. Hsp90 31 knockout targeted to male germline: a mouse model for globozoospermia. Fertil Steril. 2011;95:1475-87.

9. Jha KN, Coleman AR, Wong L, Salicioni AM, Howcroft E, Johnson GR. Heat shock protein 90 functions to stabilize and activate the testis-specific serine/threonine kinases, a family of kinases essential for male fertility. J Biol Chem. 2013;288:16308-20.

10. Volpe S, Galeati G, Bernardini C, Tamanini C, Mari G, Zambelli D, et al. Comparative immunolocalization of heat shock proteins (Hsp)-60, -70, -90 in boar, stallion, dog and cat spermatozoa. Reprod Domest Anim. 2008;43:385-92.

11. Ecroyd H, Jones RC, Aitken RJ. Tyrosine phosphorylation of HSP-90 during mammalian sperm capacitation. Biol Reprod. 2003;69:1801-7.

12. Hou ML, Huang SY, Lai YK, Lee WC. Geldanamycin augments nitric oxide production and promotes capacitation in boar spermatozoa. Anim Reprod Sci. 2008;104:56-68.

13. Zhang XG, Hu S, Han C, Zhu QC, Yan GJ, Hu JH. Association of heat shock protein 90 with motility of post-thawed sperm in bulls. Cryobiology. 2015;70:164-9.

14. Huang SY, Kuo YH, Tsou HL, Lee YP, King YT, Huang HC, et al. The decline of porcine sperm motility by geldanamycin, a specific inhibitor of heat-shock protein 90 (HSP90). Theriogenology. 2000;53:1177-84.

15. Li K, Xue Y, Chen A, Jiang Y, Xie H, Shi Q, et al. Heat shock protein 90 has roles in intracellular calcium homeostasis, protein tyrosine phosphorylation regulation, and progesterone-responsive sperm function in human sperm. PLoS One. 2014;9:e115841.

16. World Health Organization. WHO laboratory manual for the examination and processing of human semen. 5th ed. Cambridge: Cambridge University Press; 2010.

17. Bhilawadikar R, Zaveri K, Mukadam L, Naik S, Kamble K, Modi $\mathrm{D}$, et al. Levels of Tektin 2 and CatSper 2 in normozoospermic and oligoasthenozoospermic men and its association with motility, fertilization rate, embryo quality and pregnancy rate. J Assist Reprod Genet. 2013;30:513-23.

18. Sagare-Patil V, Galvankar M, Satiya M, Bhandari B, Gupta SK, Modi D. Differential concentration and time dependent effects of progesterone on kinase activity, hyperactivation and acrosome reaction in human spermatozoa. Int J Androl. 2012;35:633-44.

19. Sagare-Patil V, Modi D. Progesterone activates Janus kinase $1 / 2$ and activators of transcription 1 (JAK1-2/STAT1) pathway in human spermatozoa. Andrologia. 2013;45:178-86.

20. Sagare-Patil V, Vernekar M, Galvankar M, Modi D. Progesterone utilizes the PI3K-AKT pathway in human spermatozoa to regulate motility and hyperactivation but not acrosome reaction. Mol Cell Endocrinol. 2013;374:82-91.

21. Parte PP, Rao P, Redij S, Lobo V, D'Souza SJ, Gajbhiye R, et al. Sperm phosphoproteome profiling by ultra performance liquid chromatography followed by data independent analysis (LC$\mathrm{MS}(\mathrm{E}))$ reveals altered proteomic signatures in asthenozoospermia. J Proteomic. 2012;75:5861-71.

22. Amaral A, Paiva $\mathrm{C}$, Attardo $\mathrm{PC}$, Estanyol JM, Ballescà JL, Ramalho-Santos J, et al. Identification of proteins involved in human sperm motility using high-throughput differential proteomics. J Proteome Res. 2014;5:5670-84.

23. Siva AB, Kameshwari DB, Singh V, Pavani K, Sundaram CS, Rangaraj N, et al. Proteomics-based study on asthenozoospermia: differential expression of proteasome alpha complex. Mol Hum Reprod. 2010;7:452-62.

24. Ho HC, Suarez SS. Characterization of the intracellular calcium store at the base of the sperm flagellum that regulates hyperactivated motility. Biol Reprod. 2003;68:1590-6.

25. Baldi E, Luconi M, Muratori M, Marchiani S, Tamburrino L, Forti G. Nongenomic activation of spermatozoa by steroid hormones: facts and fictions. Mol Cell Endocrinol. 2009;24:39-46.

26. Sagare-Patil V, Modi D. Progesterone alters phosphorylation of motility associated proteins in human spermatozoa. Reprod Fertil Dev. 2016. doi:10.1071/RD15492.

27. Lu XA, Wang X, Zhuo W, Jia L, Jiang Y, Fu Y, et al. The regulatory mechanism of a client kinase controlling its own release from Hsp90 chaperone machinery through phosphorylation. Biochem J. 2014;457:171-83.

28. Wang X, Lu XA, Song X, Zhuo W, Jia L, Jiang Y, et al. Thr90 phosphorylation of Hsp $90 \alpha$ by protein kinase A regulates its chaperone machinery. Biochem J. 2012;441:387-97.

29. Wandinger SK, Suhre MH, Wegele H, Buchner J. The phosphatase Ppt1 is a dedicated regulator of the molecular chaperone Hsp90. EMBO J. 2006;25:367-76.

30. Stravopodis DJ, Margaritis LH, Voutsinas GE. Drug-mediated targeted disruption of multiple protein activities through functional inhibition of the Hsp90 chaperone complex. Curr Med Chem. 2007;14:3122-38. 